\title{
Long term trigger ventilation in neonatal respiratory distress syndrome
}

\author{
R C de Boer, A Jones, P S Ward, J H Baumer
}

\begin{abstract}
Experience of using an SLE 2000 neonatal trigger ventilator as the sole means of ventilation in 68 infants with respiratory distress syndrome was reviewed. No death or complication was seen in 22 infants with a birth weight above $1500 \mathrm{~g}$. Forty five infants under $1500 \mathrm{~g}$ birth weight including 18 infants between 23 and 28 weeks' gestation were trigger ventilated throughout. Six infants died, four of whom were under 28 weeks' gestation. Two infants under $1500 \mathrm{~g}$ birth weight sustained a pneumothorax while being ventilated. Nine of 61 infants $(15 \%)$ had radiological evidence of pulmonary interstitial emphysema, which was mild in seven infants. Intraventricular haemorrhage occurred in 10 babies under $1500 \mathrm{~g}$, four of these being grade III or IV. Twenty two $(48 \%)$ of the babies under $1500 \mathrm{~g}$ required added oxygen at 28 days. The preliminary clinical experience of this trigger ventilator suggests that it is capable of providing respiratory support from birth to extubation in even the most immature infants with respiratory distress syndrome. A controlled clinical trial is now required to compare the safety and efficacy of patient triggered ventilation with conventional neonatal ventilation.
\end{abstract}

(Arch Dis Child 1993;68:308-311)

Complications associated with ventilating premature infants for respiratory distress syndrome, including intraventricular haemorrhage, pulmonary air leaks and chronic lung disease of prematurity, occur frequently. Recent advances, such as the use of artificial surfactant, have led to a reduction in complications, but a significant morbidity still exists in the most preterm infants.

Successful trigger ventilation provides phase and rate matched ventilation such that ventilator inflation occurs only during the infant's inspiratory phase and does not extend into the infant's period of expiration. In order to achieve this a ventilator requires a sensor capable of picking up the respiratory effort of even the smallest preterm infant, and a response time short enough to ensure phase matching.

Until recently, commercially available trigger ventilators used an abdominal pneumatic capsule (the Graseby MR10) as the respiratory sensor. Mehta et al reported improved oxygenation compared with conventional ventilation as well as reduced ventilator pressure requirements using such a capsule. ${ }^{1}$ However Greenough et al pointed out the limitations of this sensor, ${ }^{2}$ and Hird and Greenough demonstrated a significantly smaller tidal volume, longer trigger delay, and lower pick up rate using the abdominal capsule compared with an airway pressure sensor just proximal to the endotracheal tube. ${ }^{3}$

Hird and Greenough demonstrated failure of successful long term trigger ventilation with an airway sensor when this was commenced early in the infant's illness, especially in those infants where there was a long trigger delay and a shorter inspiratory time $(0.24 v 0.3 \mathrm{sec}) .{ }^{4}$

The SLE 2000 neonatal ventilator is a valveless jet ventilator that operates at an airflow of 5 litres per minute and is capable of sustained inflation at very short inspiratory times. ${ }^{5}$ It uses an airway pressure sensor and has adjustable trigger sensitivity. In addition, mandatory back up ventilator inflations can be delivered at a variable rate determined by the operator.

This ventilator has been used in the neonatal intensive care unit in Plymouth for over 12 months. This report describes the technique and results of using this ventilator for preterm infants with respiratory distress syndrome.

\section{Patients and methods}

The records of all infants ventilated between 1 January and 31 December 1991 for respiratory distress syndrome were reviewed and details of complications noted. All infants under 32 weeks' gestation had head ultrasound scans performed by a consultant radiologist experienced in neonatal ultrasound during the first week of life and again before discharge.

Artificial surfactant (Exosurf, Wellcome) was administered to all infants ventilated for respiratory distress syndrome in a dose of 5 $\mathrm{ml} / \mathrm{kg}$, with a second dose if they still required ventilation at 24 hours. Ethamsylate was given to infants under 32 weeks' gestation for four days. From August 1991, Intralipid (Kabi, Pharmacia) was withheld until infants had been successfully weaned from ventilation. Infants under 30 weeks' gestation were started on theophylline at the onset of ventilation.

Blood pressure was monitored via an indwelling peripheral or umbilical arterial line. Cardiovascular support in the form of plasma and inotropes was given as required, aiming to keep the mean arterial blood pressure above $30 \mathrm{~mm} \mathrm{Hg}$.

Arterial blood gases were checked four hourly and within an hour of making changes to ventilation. Arterial carbon dioxide tension
Freedom Fields Hospital,

Accepted 14 September 1992 
was maintained in the range of 4.0 to $6.7 \mathrm{kPa}$ during the first 72 hours, but was subsequently allowed to rise further as long as the arterial $\mathrm{pH}$ was maintained above $7 \cdot 25$. Blood volume expanders and buffer (THAM) were used in the management of metabolic acidosis. Paralysis was not used at any stage. Any infant who was distressed or uncomfortable during trigger ventilation was given an intravenous bolus of morphine, $0.05 \mathrm{mg} / \mathrm{kg}$, repeated at intervals of one to two hours. In practice most infants on trigger ventilation appeared comfortable and relaxed and routine use of morphine or sedatives was not required. The humidifier was set to produce an airway temperature proximal to the incubator of approximately 36.5 to $37 \cdot 0^{\circ} \mathrm{C}$. The airway filter next to the ventilator jet manifold was changed daily.

Trigger ventilation was commenced with an inspiratory time of between 0.2 and $0.3 \mathrm{sec}-$ onds. The trigger sensitivity was increased until direct visual observation of the infant's respiratory movements while listening to the ventilator in trigger mode revealed synchronous ventilation. During synchronous ventilation the infant's respiratory efforts looked comfortable and ventilator inflation occurred at each breath. If during synchronous ventilation chest movements looked laboured, the peak inspiratory pressure was increased and the inspiratory time reduced stepwise to 0.2 seconds until the infant appeared more comfortable. The back up rate was initially set at 40 breaths per minute, checking that this was at least 20 breaths per minute below the spontaneous respiratory rate of the infant.

During ventilation, before any adjustment to the ventilator settings, the staff were taught to look for visual evidence of synchronous ventilation. Oxygenation was increased by increasing inspired oxygen concentrations or peak inspiratory pressure. If these measures failed, the inspiratory time was increased up to a maximum of 0.5 seconds. Arterial carbon dioxide tension levels were reduced by increasing peak inspiratory pressures or reducing inspiratory times to a minimum of $0.2 \mathrm{sec}-$ onds. Positive end expiratory pressure was maintained throughout at a level of 4 to $5 \mathrm{~cm}$ $\mathrm{H}_{2} \mathrm{O}$.

The back up rate was reviewed every four hours based on the infant's average respiratory rate over the previous four hours, aiming to keep the back up ventilator rate approximately 20 breaths per minute below that of the infant's spontaneous respiratory rate. At no stage did the ventilator need to be changed to mandatory ventilation mode. If a period of intermittent positive pressure ventilation was thought to be required (as in an apnoeic very immature infant) then the back up rate was set at the desired level, in patient trigger mode, thus avoiding active expiration against the ventilator.

Infants were weaned from trigger ventilation by reducing peak inspiratory pressures. The infant's respiratory pattern was observed. Comfortable synchronous respirations and satisfactory arterial blood gases were taken to indicate adequate ventilator support. Peak inspiratory pressures were gradually reduced to $8 \mathrm{~cm} \mathrm{H}_{2} \mathrm{O}$ with a positive end expiratory pressure of $4 \mathrm{~cm} \mathrm{H}_{2} \mathrm{O}$. When an infant was comfortable on these pressures the infant was extubated into a headbox or onto nasal continuous positive airway pressure.

If it was not possible to get the infant to trigger the ventilator, the back up rate was reduced, the inspiratory time reduced, and the sensitivity adjusted. If these measures failed to achieve synchronous ventilation, the possibility of a dislodged or blocked tube was considered.

The following nursing observations were undertaken hourly while the infant was ventilated: the number of triggered breaths in the previous minute (read from the ventilator), the number of untriggered breaths (counted on the ventilator) over one minute, and the ventilator back up rate.

Serial chest $x$ ray films taken in the first 10 days were assessed for signs of pulmonary interstitial emphysema (PIE) by one of us (AJ) using the scoring system described by Greenough et al. ${ }^{6}$ A maximum PIE score for each lung of 27 points is allotted to the most severe changes.

\section{Results}

During 1991, 68 babies with respiratory distress syndrome received trigger ventilation via the SLE 2000 as their only form of ventilatory support. A further 15 babies ventilated with the SLE 250 due to unavailability of the SLE 2000 have been excluded from further analysis.

Twenty two babies with birth weights greater than $1500 \mathrm{~g}$ were trigger ventilated throughout their illnesses. The median gestational age was 32 weeks (range 30 to 37 weeks) with a median birth weight of $1865 \mathrm{~g}$ (range 1530-3220 g). The duration of ventilation ranged from less than one to six days. None of the babies died. No infant developed a pneumothorax while being ventilated, nor did any required added oxygen at 28 days. There was no case of intraventricular or pulmonary haemorrhage.

Forty six babies with birth weights less than $1500 \mathrm{~g}$ were trigger ventilated for respiratory distress syndrome. One infant with trisomy 18 and relatively mild respiratory distress syndrome was excluded from further analysis. The median gestation of the remaining 45 babies was 29 weeks (range 23-32 weeks) and median birth weight was $1125 \mathrm{~g}$ (range 545-1493 g). The duration of ventilation ranged from less than one to 28 days, with a median of five days.

Six infants died (12\%). Two infants (4\%) developed a pneumothorax while being ventilated; a further five infants in this group developed pneumothoraces either before, or in one infant, some considerable time after being ventilated. Intraventricular haemorrhages occurred in 10 babies (22\%), with four of these being grade III or IV. No infant developed periventricular leucomalacia in 
the absence of intraventricular haemorrhage. Pulmonary haemorrhages were seen in four babies (9\%). Twenty two of the 45 babies needed added oxygen at 28 days, including all but two infants below 28 weeks' gestation. Ten babies with birth weights under $1500 \mathrm{~g}$ were in added oxygen at 36 weeks' postconceptional age, and four infants were discharged from hospital needing added oxygen.

Among the 19 babies under 28 weeks' gestation who were ventilated for respiratory distress syndrome, there were four deaths $(22 \%)$, all occurring within three days of birth. Survivors include a baby of 23 weeks' gestation with a birth weight of $605 \mathrm{~g}$ who was trigger ventilated for 27 days.

Hourly nursing observations of the number of breaths triggered over one minute (read from the ventilator) and the number of untriggered ventilator breaths (counted over one minute) were analysed for the first 72 hours in 16 babies. The mean and range of these observations can be seen in the figure. Mean numbers of triggered and untriggered breaths did not vary significantly with gestation.

Two infants of 25 and 27 weeks' gestation had significant periods of apnoea. The baby of 25 weeks' gestation was triggering well at rates of approximately 60 to 70 breaths per minute until 23 hours of age when a pneumothorax developed; recovery of successful ventilator activation occurred by 48 hours of age. The second infant, weighing $700 \mathrm{~g}$ at 27 weeks' gestation took two hours after ventilation commenced to activate the trigger mechanism; breath activation continued up to the time of death at 60 hours of age from respiratory failure, with peak inspiratory pressures of $40 \mathrm{cms} \mathrm{H}_{2} \mathrm{O}$.

Chest $x$ ray films were available for 61 of the 68 infants. No evidence of PIE was seen in 52 infants (85\%); PIE was mild in all but two infants. These were twins born at 25 weeks with maximum PIE scores of 26 and 30 within the first 24 hours; both died within 48 hours of birth with respiratory failure. The average of the worst PIE scores in the remaining seven babies was $10 \cdot 2$, with a maximum of 14 .

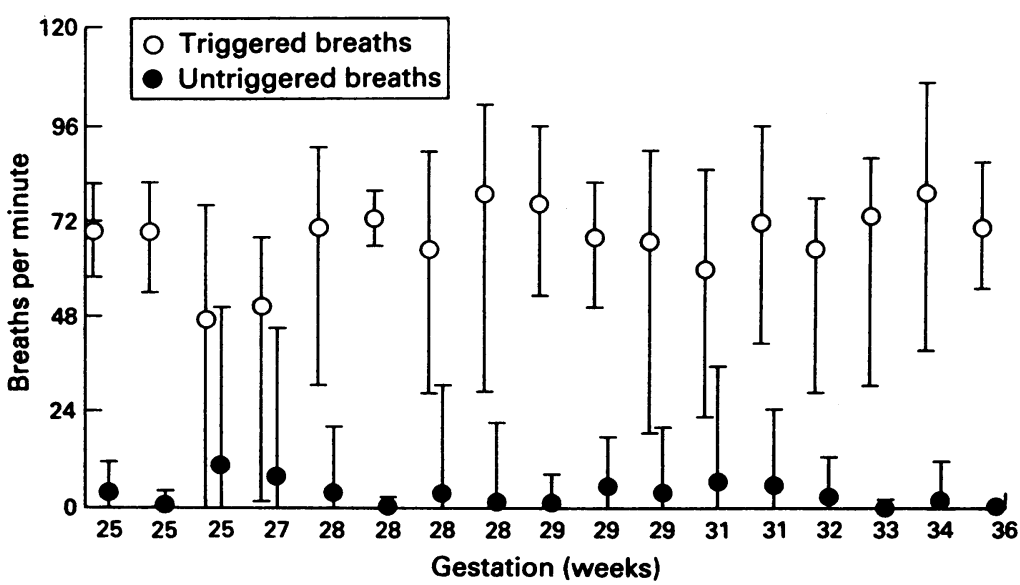

Triggered and untriggered breaths in the first 72 hours after birth; results are mean and range.

\section{Discussion}

We have found the SLE 2000 neonatal ventilator to be capable of providing synchronous patient triggered ventilation for premature infants with respiratory distress syndrome from birth to extubation in even the most immature infants. The observations of spontaneous respirations and ventilator backup rates confirm that the infants were receiving trigger ventilation rather than intermittent mandatory ventilation for most of the time.

This experience is at variance with the statement of Greenough and Milner that patient trigger ventilation is frequently unsuccessful in the most immature infants. ${ }^{7} \mathrm{We}$ believe that the main reasons for this are the performance characteristics of the jet ventilator, the ability to vary the back up ventilation rate and the sensitivity of the airway pressure sensor.

A number of devices have been used as triggers sensors, including an abdominal pneumatic capsule, ${ }^{1}$ an oesophageal balloon, ${ }^{8}{ }^{9}$ a pneumotachograph ${ }^{2}$ and an airway pressure sensor. $^{3}$ The abdominal capsule and oesophageal balloon both have the disadvantage of requiring skill to achieve optimal placement, with the possibility of either movement artefact or peristalsis modifying the signal. Neither is capable of recognising obstructed breathing. The sensitivity of the oesophageal balloon may decline over time as secretions accumulate. A pneumotachograph gives information on tidal volume and only triggers with unobstructed breaths; however, it increases the dead space and may be affected in long term use by condensation. An airway pressure sensor only triggers with unobstructed breaths and does not increase dead space, but gives no information on tidal volume.

The sensitivity of the airway pressure sensor is inversely proportional to the airflow through the ventilator tubing. With the flow rate of 5 litres per minute through the SLE 2000 ventilator the sensor is sufficiently sensitive to pick up the respiratory efforts of even the most preterm infants. We find it important to be able to adjust the trigger sensitivity for each individual baby, although it is unusual to find problems with too high a sensitivity.

Our experience with patient triggered ventilation using abdominal capsule sensor was that infants would frequently become 'tired' manifested by deteriorating blood gases or apnoea. The variable back up breath facility on the SLE 2000 enabled us to continue ventilating infants in trigger mode despite periods of apnoea. The routine administration of theophylline to babies less than 30 weeks' gestation may further help to reduce the problem of apnoea.

The valveless design of the ventilator permits a rapid increase in inspiratory pressure and an inspiratory plateau, with consequent improved oxygenation at very short inspiratory times. This, together with the very short delay time and great sensitivity of the trigger sensor together result in better phase matching with the baby's respirations, reducing the risk of active expiration against the ventilator. 
The pneumothorax rate in the first year of using this ventilator was extremely low. The use of artificial surfactant clearly contributed to the rarity of this complication. The rate of pneumothorax in infants with respiratory distress syndrome was higher when not being ventilated, and we suggest that the ventilation technique may well have helped to keep the pneumothorax rate low.

Severe pulmonary interstitial emphysema was seen rarely in this high risk group of infants. The effects of artificial surfactant cannot be assessed in this uncontrolled study. The $15 \%$ rate of pulmonary interstitial emphysema was slightly lower than in the series of Greenough et al where 40 of 210 preterm infants ventilated for respiratory distress syndrome had radiological evidence of PIE. ${ }^{6}$ The average of the worst PIE scores in our study was very much lower (14 v 23) than that reported by Greenough et al, and only two infants in our series had maximum scores above 14. Unlike Greenough et al we found PIE only in the more immature infants.

It is difficult to interpret the $48 \%$ incidence of oxygen dependency at 28 postnatal days in the infants under $1500 \mathrm{~g}$ birth weight. Significant differences in the incidence of oxygen dependence at 28 days were found between eight centres in the United States, ${ }^{10}$ but the reasons for these differences remain uncertain. Early use of Intralipid in the neonatal unit was reduced during the period of the study after reports of an association with an increased risk of chronic lung disease. ${ }^{11} 12$ It has been suggested that the requirement for added oxygen at 36 weeks' gestation may be a better guide to later pulmonary problems in infants with a birth weight of less than $1500 \mathrm{~g} .{ }^{13}$ In our series 10 infants $(21 \%)$ of those with birth weights below $1500 \mathrm{~g}$ needed added oxygen at 36 weeks' gestation, and four infants still needed added oxygen at the time of discharge home.

The finding of pulmonary haemorrhage in four infants is of concern, especially as one of the infants subsequently died. It is possible that the pulmonary haemorrhage contributed to the baby's death.

Although the evidence is purely anecdotal, we believe that trigger ventilation has other benefits. The medical and nursing staff find the technique of ventilation easy to master, and believe that it gives babies of all gestations a smoother period of ventilation. Mature infants appear more settled than on intermittent mandatory ventilation.

The relatively low rates of immediate complications are encouraging, but we emphasise that no inference can be drawn from our experience as to the efficacy of this form of ventilation as compared with conventional neonatal ventilation. Advances in neonatal care, including the introduction of artificial surfactant and the improved delivery of intensive care, invalidate comparison with historical controls.

We believe that there is now a need to undertake a randomised controlled trial to compare the safety and efficacy of trigger ventilation with conventional ventilation. However, large numbers of infants would need to be enrolled. In one recent controlled trial of conventionally ventilated infants with respiratory distress syndrome, $74(12 \%)$ of 615 babies receiving artificial surfactant developed extrapulmonary air leak. ${ }^{14} \mathrm{We}$ found a $4 \%$ rate of extrapulmonary air leak in our series. In order to have an $80 \%$ chance of detecting a difference of this magnitude, significant at the 5\% level, 200 infants would need to be enrolled into each arm of a controlled trial.

In conclusion, this is the first report of long term trigger ventilation in premature infants of all gestations throughout their period of ventilatory support for respiratory distress syndrome. A large, randomised controlled study is now required to establish further the place of patient triggered ventilation.

1 Mehta A, Callan K, Wright BM, Stacey TE. Patient-triggered ventilation in the newborn. Lancet 1986;ii:17-9.

2 Mitchell A, Greenough A, Hird M. Limitations of patien triggered ventilation in neonates. Arch Dis Child 1989;64: triggered

3 Hird M F, Greenough A. Comparison of triggering systems for neonatal patient triggered ventilation. Arch Dis Child 1991;66:426-8.

4 Hird M F, Greenough A. Causes of failure of neonatal patient triggered ventilation. Early Hum Dev 1990;23: 101-8.

5 Chan KN, Chakrabarti MK, Whitwam JG, Silverman M. Assessment of a new valveless infant ventilator. Arch Dis Child 1988;63:162-7.

6 Greenough A, Dixon AK, Roberton NRC. Pulmonary interstitial emphysema. Arch Dis Child 1984;59:1046-51.

7 Greenough A, Milner AD. Respiratory support using patient triggered ventilation in the neonatal period. Arch Dis Child 1992;67:69-71.

8 Greenough A, Greenall F. Patient triggered ventilation in premature neonates. Arch Dis Child 1988;63:77-8.

9 Gremature neonates. Arch Dis Child 1988;63:77-8. Greenough A, Pool J. Neonatal patient
tion. Arch Dis Child 1988;63:394-7.

10 Avery ME, Tooley WH, Keller JB, et al. Is chronic lung disease in low birth weight infants preventable? A survey of eight centers. Pediatrics 1987;79:26-30.

11 Hammerman C, Aramburo MJ. Decreased lipid intake reduces morbidity in sick premature neonates. $\mathcal{f}$ Pediat 1988;113:1083-8.

12 Cooke RWI. Factors associated with chronic lung disease in preterm infants. Arch Dis Child 1991;66:776-9.

13 Shennan AT, Dunn MS, Ohlsson A, Lennox K, Hoskins EM. Abnormal pulmonary outcomes in premature infants: prediction from oxygen requirement in the neonatal period. Pediatrics 1988;82:527-32.

14 Long W, Corbet A, Cotton R, et al. A controlled trial of synthetic surfactant in infants weighing $1250 \mathrm{~g}$ or more with respiratory distress syndrome. N Engl f Med 1991; 325: 1696-703. 